\title{
Applying the PRISMA method for obtaining systematic reviews of occupational safety issues in literature search
}

\author{
Alina Trifu' ${ }^{*}$, Eduard Smîdu ${ }^{2}$, Daniel Onuţ Badea ${ }^{1}$, Eugenia Bulboacă ${ }^{2}$, Vergilică \\ Haralambie $^{2}$ \\ ${ }^{1}$ INCDPM Institutul National de Cercetare-Dezvoltare pentru Protecțía Muncii” Alexandru Darabont” \\ București, B-dul Ghencea 35A, România \\ ${ }^{2}$ University Politehnica of Bucharest, Splaiul Independenţei nr. 313, Sector 6, Romania
}

\begin{abstract}
This paper presents the work of $5 \mathrm{PhD}$ students in elaboration of their doctoral thesis, especially for the first part of the thesis, namely the state of the art. The method used was The Preferred Reporting Items for Systematic reviews and Meta-Analyses (PRISMA). Using the chart flow and the list of items of this method, a literature search was conducted in Science Direct Freedom Collection, Elsevier database, Web of Science Core Collection, Springer Link Journals. In this paper are given 2 examples of systematic reviews that were obtained using PRISMA method.
\end{abstract}

\section{Introduction}

Systematic reviews are essential not only for researchers, policy makers and other decision makers but also for students, who without them, would be confronted by an overwhelming volume of research on which to base the "state of the art" chapter in their thesis. The methods and results of systematic reviews should be reported in sufficient detail to allow users to assess the trustworthiness and applicability of the review findings. For this paper it was used The Preferred Reporting Items for Systematic reviews and Meta-Analyses (PRISMA). The PRISMA has been designed primarily for systematic reviews of studies that evaluate the effects of health interventions, irrespective of the design of the included studies. However, the checklist items are applicable to reports of systematic reviews evaluating other non-health-related interventions (for example, safety and health, occupational safety), and many items are applicable to systematic reviews with objectives other than evaluating interventions [1]. The Preferred Reporting Items for Systematic reviews and Meta-Analyses (PRISMA) statement, published in 2009 (hereafter referred to as PRISMA 2009) [1, 2] was designed to help authors prepare transparent accounts of their reviews, and its recommendations have been widely endorsed and adopted.

\footnotetext{
* Corresponding author: alinatrifu@yahoo.com
} 


\section{Method}

The Preferred Reporting Items for Systematic reviews and Meta-Analyses PRISMA is published as a suite of three papers: a statement paper, the PRISMA abstract checklist, and the flow diagram [3].

\subsection{Starting the systematic review}

Because we were carried out the review as a members of a wider team, we consulted the other members of our team regularly in order to prevent misunderstandings. Different people may use the terminology in different ways depending on their geographical location or profession. We also kept regular contact with our supervisor is worthwhile, in order to benefit from her professional expertise. We followed this general pointers:

- Limits: Once the topic has been clearly defined, we considered whether we want to limit the search by language or date of publication. This may be helpful when the topic is fairly new, but applying limits can miss out definitive older articles.

- Defining the search terms: The best way to do this was to run a couple of wide searches on the most commonly used databases in our subjects. We found some relevant articles and read through them highlighting key words or phrases. Then we used them as the basis for our search strategy. We made a list of the core terms and any synonyms and spelling variations. At this stage we have discussed these terms with our project team or supervisor.

- Thesaurus terms: The most precise way to search any database was to use the words included in the thesaurus for the individual database. As new articles were added to a database, they were indexed using a list of approved keywords or thesaurus. This is why we always searched one database at a time - remembering that thesauri vary from database to database. Even natural language words can vary in the way they are used. Using this type of search produced very focused results.

- Natural language searching: because the research protocol required to widen out the search to ensure that is not miss out any research and many grey literature sources and search engines do not use thesauri, we used a combination of terms, alternative keywords when searching.

\subsection{Filling in the PRISMA flowchart}

\subsubsection{Step 1: Preparation}

To complete the PRISMA diagram we printed out a copy of the diagram to use alongside our searches. It is most efficient to search databases individually, so we printed out a copy for each database searched, plus a copy for the totals. Because we used this system for a more advanced assignment, such as a thesis, we asked our supervisor whether she would like us to follow this system, or to specify totals for each individual database in our final PRISMA diagram.

\subsubsection{Step 2: Doing the database search}

For each database we entered each key search term individually. This included all our search terms. We combined all the search terms in the different combinations using Boolean operators like AND OR as appropriate. We applied all our limits (such as years of search, full-text only, English language only and so on). Once all search terms have been combined and we have applied all relevant limits, we obtained a number of records or 
articles and entered this in the top left box of the PRISMA flow chart for each database. Because we have searched databases individually, we added all the 'records identified' up and fill this total number in the PRISMA flow diagram. This process of adding up the number of records in individual database searches to a total was repeated at each step.

\subsubsection{Step 3: Additional sources}

For the articles identified through other sources than databases (like manual searches through reference lists of articles found, or search engines like Google Scholar), we entered the total number of records in the box on the top right of the flow diagram.

\subsubsection{Step 4: Remove all duplicates}

To avoid reviewing duplicate articles, it was removed manually any articles that appear more than once by going through all the records or articles found in the database. The number of records left after the removal of the duplicates was entered in the second box from the top.

\subsubsection{Step 5: Screening articles}

The next step was to add in the number of articles that we have screened. This is the same number as we have entered in the duplicates removed box

\subsubsection{Step 6: Screening - Excluded articles}

In this step we screened the titles and abstracts for articles which were relevant to our research question. Any articles that appeared to help us provide an answer to our research question it was included. The number of articles excluded based on this screening process it was recorded in the appropriate box (next to the total number of screened records) with a short reason for excluding these articles.

\subsubsection{Step 7: Eligibility}

It was subtracted the number of excluded articles following the screening phase (step 6) from the total number of records screened (step 5) and entered this number in the box titled "Full-text articles assessed for eligibility". The full text for these articles was reviewed for eligibility.

\subsubsection{Step 8: Eligibility - Records excluded}

We reviewed all full-text articles for eligibility to be included in the final review. At this stage we checked in our guidelines and with our supervisor, how many articles we should be left with. The number of articles that were excluded at this point was entered in the box titled: Full text articles excluded and we write in a short reason for excluding the articles (it may be the same reason used for the screening phase).

\subsubsection{Step 9: Included}

The final step was to subtract the number of excluded articles or records during the eligibility review of full-texts (step 8) from the total number of articles reviewed for 
eligibility (step 7). The number obtained may varied depending on the type of assignment and it was entered in the final box. The PRISMA flow diagram was now completed and can now be included in the results section of the assignment.

\section{Results and discussion}

We present two examples of applying PRISMA method.

\subsection{Example 1}

For the thesis "Theoretical and experimental research on the analysis of noxious emissions in the case of welding in shielding gases" a literature search was conducted in ScienceDirect Freedom Collection, Elsevier database, Web of Science - Core Collection, Clarivate Analytics, Scopus, SpringerLink Journals. Keywords such as welding fume, airborne particles from welding operations, welding aerosol, chemical analysis of welding fume particle, welding fumes exposure were used to retrieve relevant studies which explicitly reported on occupational risks related to welding. The searches were limited to English and Romanian languages only. The PRISMA checklist was used to validate the research process [2].

The titles and abstracts of the search results were screened independently by all authors with discrepancies discussed and resolved.

Articles were eligible for full-text screening if the title and/or abstract mentioned welding fume, airborne particles from welding operations, welding aerosol, chemical analysis of welding fume particle, welding fumes exposure. Full-texts were screened for inclusion by all authors disagreements resolved by discussion. Articles were included in this systematic review if they reported on occupational risks related to noxious emissions in welding with shielding gases. Research studies that made use of previously collected or administrative data were also acceptable if they satisfied other criteria. Studies were excluded if they were published before 1995. [11][12]

Un exemple of exclusion was the fumes resulted from other then MIG and MAG welding processes: Sowards JW, Ramirez AJ, Dickinson DW, Lippold JC (2010) Characterization of welding fume from SMAW electrodes [14] [15] 


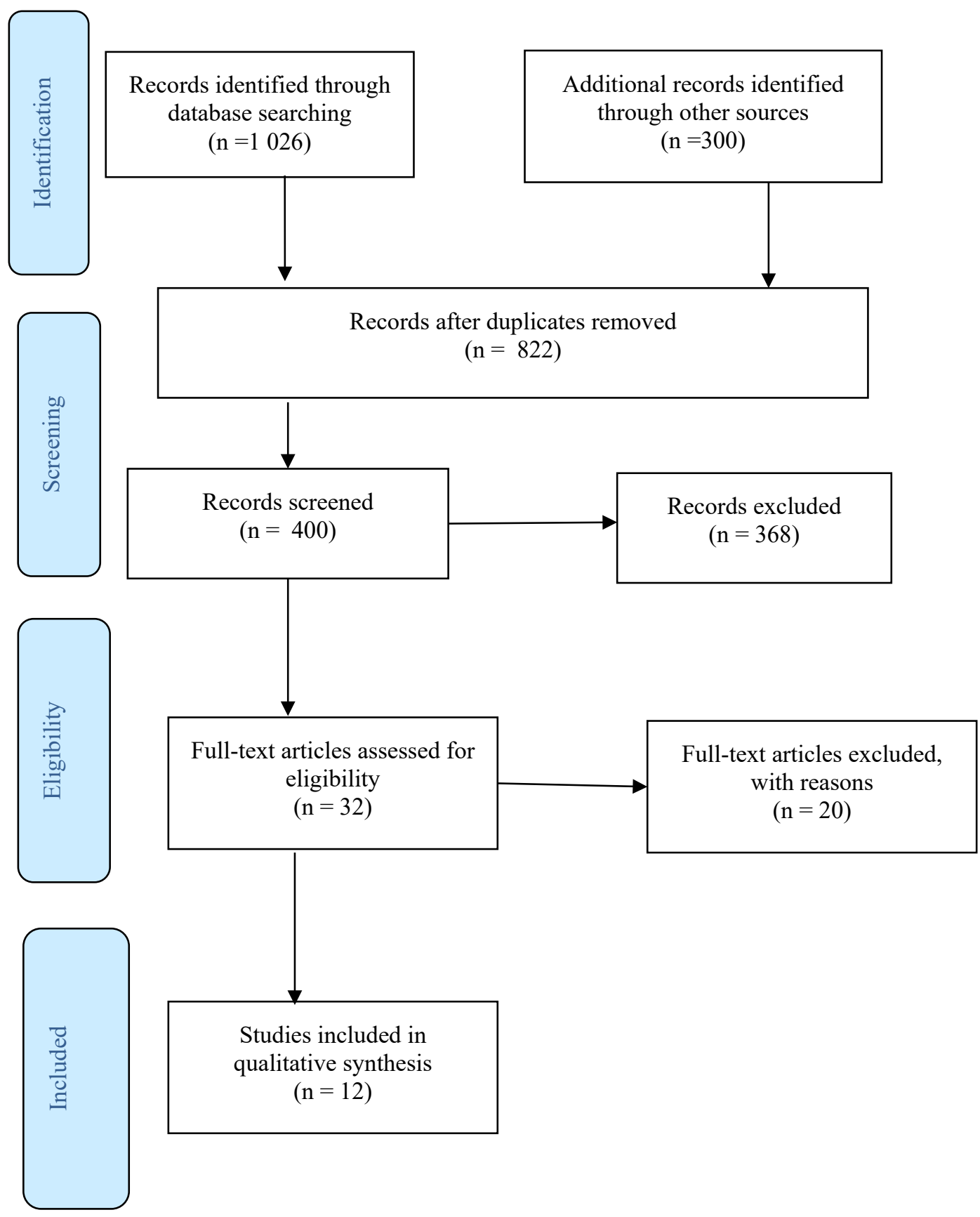

Fig. 1. PRISMA flow diagram for example 1 


\subsection{Example 2}

For the thesis "Occupational risks assessment and mitigation systems" a literature search was conducted in ScienceDirect Freedom Collection, Elsevier database, Web of Science Core Collection, Clarivate Analytics, Scopus, SpringerLink Journals. Keywords such as occupational safety, health and safety evaluation risks, methods of risk assessment, risk mitigation were used to retrieve relevant studies which explicitly reported on occupational risks related to welding. The searches were limited to English and Romanian languages only. The PRISMA checklist was used to validate the research process [2].

The titles and abstracts of the search results were screened independently by all authors with discrepancies discussed and resolved.

Articles were eligible for full-text screening if the title and/or abstract mentioned occupational safety, health and safety evaluation risks, methods of risk assessment, risk mitigation. Full-texts were screened for inclusion by all authors disagreements resolved by discussion. Articles were included in this systematic review if they reported on methods of risk assessment for occupational risks and risks mitigation. Research studies that made use of previously collected or administrative data were also acceptable if they satisfied other criteria. Studies were excluded if they were published before 2000 . 


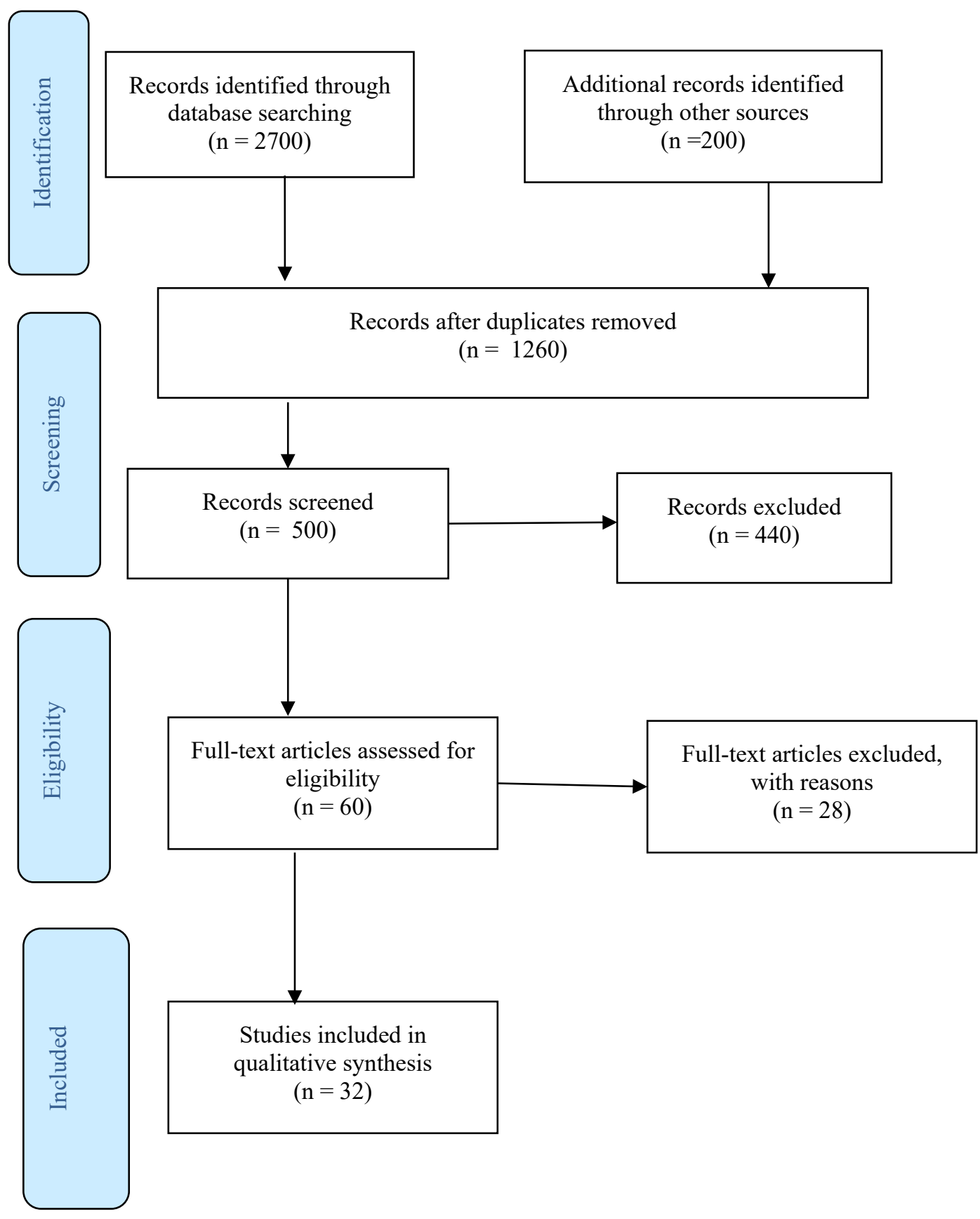

Fig. 2. PRISMA flow diagram for example 2 


\section{Conclusions}

The Preferred Reporting Items for Systematic reviews and Meta-Analyses (PRISMA) was a great help for authors to prepare transparent accounts of their reviews. Systematic reviews are essential not only for researchers, policy makers and other decision makers but also for students, who without them, would be confronted by an overwhelming volume of research on which to base the "state of the art" chapter in their thesis. With the help of PRISMA the methods and results of systematic reviews can be reported in sufficient detail to allow users to assess the trustworthiness and applicability of the review findings. For this paper it was used The Preferred Reporting Items for Systematic reviews and Meta-Analyses (PRISMA) published in 2009. The PRISMA has been designed primarily for systematic reviews of studies that evaluate the effects of health interventions, irrespective of the design of the included studies. However, the checklist items were applicable to reports of systematic reviews evaluating other non-health-related interventions (for example, safety and health, occupational safety), and many items were applicable to systematic reviews with objectives other than evaluating interventions.

\section{References}

1. The PRISMA 2020 statement: an updated guideline for reporting systematic reviews [Online]. Available https://www.bmj.com/content/372/bmj.n71. [Accessed 22 march 2021]

2. The PRISMA 2009 checklist. [Online]. Available https://www.bmj.com/content/372/bmj.n71. [Accessed 22 march 2021]

3. The PRISMA 2020 statement: an updated guideline for reporting systematic reviews. Submitted 2020 [Online]. Available https://www.bmj.com/content/372/bmj.n71. [Accessed 22 march 2021]

4. N. Floros, Weld World 62, 311-316 (2018)

5. EN ISO 15011-2:2009, Health and safety in welding and allied processes. Laboratory method for sampling fume and gases. Determination of the emission rates of carbon monoxide (CO), carbon dioxide $\left(\mathrm{CO}_{2}\right)$, nitrogen monoxide $(\mathrm{NO})$ and nitrogen dioxide $\left(\mathrm{NO}_{2}\right)$ during arc welding, cutting and gouging

6. EN ISO 15011-3:2009, Health and safety in welding and allied processes. Laboratory method for sampling fume and gases. Determination of ozone emission rate during arc welding

7. EN ISO 15011-5:2011, Health and safety in welding and allied processes. Laboratory method for sampling fume and gases. Identification of thermal-degradation products generated when welding or cutting through products composed wholly or partly of organic materials using pyrolysis-gas chromatography-mass spectrometry

8. A.T. Zimmer, P. Biswas, J Aerosol Sci Aug, 32 (2011)

9. B. Moroni, C. Viti, J Aerosol Sci, 40 (2009)

10. J Dasch, J D'Arcy (2008), J Occup Environ Hyg 5 (1975)

11. M Kobayashi, S Maki, Y Hashimoto, T Suga, Welding in the World, 16 (1978)

12. C.N. Gray, P.J. Hewitt, P.R.M. Dare Welding and Fabrication J, October, 393-397(2002)

13. JW Sowards, JC Lippold, DW Dickinson, AJ Ramirez, Weld J, 87 (2008)

14. JW Sowards, AJ Ramirez, DW Dickinson, JC Lippold, Weld J, 89 (2010) 